\title{
Effects of Repeated Acquisitions and Extinctions on Response Rate and Pattern
}

\author{
Paulo Guilhardi, Linlin Yi, and Russell M. Church \\ Brown University
}

\begin{abstract}
The procedure developed by R. A. Rescorla (2002) was used to study the effects of repeated acquisitions and extinctions of head entry responses into a food cup by rats. In each of 420 -session phases, food was delivered at the end of particular 30-s auditory and visual stimuli, but not at the end of different 30-s auditory and visual stimuli. Based on response rates to individual stimuli and compound stimuli, the increase in response rate in acquisition occurred more rapidly than the decrease in extinction. Acquisition, but not extinction, occurred faster after successive transitions between acquisition and extinction. Temporal gradients of responding developed during acquisition and remained during extinction. Conclusions based on mean response rate, temporal gradients, and transfer tests were consistent.
\end{abstract}

Keywords: extinction, memory, fixed interval, duration, reinforcement rate

During acquisition, when a stimulus is followed by food delivery, rats increase their rate of head entry responses into the food cup in the presence of the stimulus relative to the absence of the stimulus. The absolute response rate is determined by such factors as the mean reinforcement rate in the presence of the stimulus, the amount of training, and the salience of the stimuli. The effects of reinforcement rate on response rate, and reinforcement time on response pattern, occur both in classical procedures with responseindependent delivery of reinforcement and instrumental procedures with response-dependent delivery of reinforcement (Guilhardi \& Church, 2005; Guilhardi, Keen, MacInnis, \& Church, 2005; Kirkpatrick \& Church, 2003).

During extinction, when a stimulus is no longer followed by food delivery, there is a substantial reduction in response rate without a loss of the learned associations (Rescorla, 1996, 2001a). In a temporal conditioning procedure, extinction also results in a substantial reduction in response rate without loss of the response pattern (Guilhardi \& Church, in press; Ohyama, Gibbon, Deich, \& Balsam, 1999).

The standard linear operator models of learning (Bush \& Mosteller, 1951; Estes, 1950) use different asymptotic levels and different learning rate parameters for acquisition and extinction of observed behavior. Typically, the rate of acquisition of the behavior is greater than the rate of extinction of the behavior, but, as Rescorla (2002) emphasized, this is not equivalent to a claim that

Paulo Guilhardi, Linlin Yi, and Russell M. Church, Department of Psychology, Brown University.

The primary data are archived for further analysis (http://dx.doi.org/ 10.1037/0097-7403.32.3.322.supp). They consist of the times of stimulus onset and termination, of head entry and withdrawal responses, and food delivery for each rat on each session. See the readme.doc file for details.

This work was supported by National Institute of Mental Health Grant MH44234 to Brown University.

Correspondence concerning this article should be addressed to Paulo Guilhardi, Department of Psychology, Box 1853, Brown University, Providence, RI 02912. E-mail: Paulo_Guilhardi@brown.edu the rate of acquisition of associative strength is greater than the rate of extinction of associative strength. This is because associative strength is assumed to have an ordinal relationship to observed behavior, but not necessarily a linear one (Rescorla \& Wagner, 1972). For inferences about changes in the amount of associative strength of individual stimuli, Rescorla (2000, 2001b) developed a transfer test technique that used compound stimuli and did not require a mapping between the magnitude of associative strength and response rate.

In a report of five experiments that used this technique, Rescorla (2002) found that the rate of associative change was greater during acquisition than during extinction. The present experiment is a replication and extension of the first of these experiments. It includes an analysis of the acquisition and extinction of the response rate and temporal gradients of responding, the asymptotic gradients of responding after acquisition and extinction, as well as the rate of head entries during compound stimuli. The focus of this article is on a comparison of the conclusions based upon an analysis of the responding in the presence of the elementary stimuli and the stimulus compounds during repeated acquisitions and extinctions.

\section{Method}

\section{Animals}

Twenty-four male Sprague-Dawley rats (Taconic Laboratories, Germantown, NY) were used in the experiment. The rats were housed individually in a colony room on a 12:12 light:dark cycle (lights off at 8:30 a.m.). Dim red lights provided illumination in the colony room and the testing room. The rats were fed a daily ration that consisted of 45-mg Noyes pellets (Improved Formula A) that were delivered during the experimental session, and an additional $15 \mathrm{~g}$ of FormuLab 5008 food given in the home cage after the daily sessions. Water was available ad libitum in both the home cages and experimental chambers. Twelve of the rats were from an experiment in which they had food delivered independently of responding at the end of fixed and/or random durations that were signaled with noise and/or light stimuli; the other 12 rats were from another experiment in which they had been reinforced and extinguished on a 
multiple cued temporal discrimination task during which noise, houselight, and clicker were each associated with one of three fixed intervals (30-, 60-, and 120-s) that were presented intermixed within a session. Training for the present experiment began when the rats were 158 days and 621 days old for the two sets of animals, respectively.

\section{Apparatus}

The 12 boxes $(25 \times 30 \times 30 \mathrm{~cm})$ were located inside ventilated, noise-attenuating enclosures $(74 \times 38 \times 60 \mathrm{~cm})$. Each box was equipped with a food cup and a water bottle. Four stimuli, referred to as noise, clicker, houselight, and flashing light, were generated from modules from Med Associates, St. Albans, VT. The noise was a 70-dB white noise, with an onset rise time and termination fall time of $10 \mathrm{~ms}$, that was generated by an audio amplifier (Model ANL-926). The clicker (Model ENV-135M) was a small relay mounted on the outside of the box that was used to produce an auditory click at a rate of 2 per second. The houselight was a diffused houselight (Model ENV-227M) rated to illuminate the entire chamber over 200 lux at a distance of 3 in $(7.62 \mathrm{~cm})$. The flashing lights were two 100-mA stimulus lights (ENV-221M) with 1-in. (2.54-cm) white lens, mounted on the opposite wall from the houselight, that flashed simultaneously at a rate of 1 per second.

A pellet dispenser (Model ENV-203) delivered 45-mg Noyes pellets (Improved Formula A) into the food cup on the front wall. Each head entry into the food cup was detected by an LED photocell. A water bottle was mounted outside the box; water was available through a tube that protruded through a hole in the back wall of the box. Two Gateway Pentium III/500 computers running the Med-PC Medstate Notation, Version 2.0 (Tatham \& Zurn, 1989), controlled experimental events and recorded the time at which events (stimuli, responses, and reinforcers) occurred with 2-ms resolution.

\section{Procedure}

The procedure was similar to the one used by Rescorla (2002, Experiment 1), but with 20 (rather than 2) sessions in each phase of reversal training, 3 (rather than 1) successive reversal phases, and a fourfold increase in the number of presentations of compound stimuli in a phase.

Prior to each session, there was a test of each of the stimuli (noise, clicker, houselight, and flashing light), the recording of head entries into the food cup, and the delivery of food pellets. There was one pellet in the food tray at the beginning of each session. The experiment consisted of 80 sessions, during which one of the stimuli was presented for $30 \mathrm{~s}$ followed by a variable intertrial interval in which no stimulus was presented. The variable intertrial interval was composed of a minimum of a fixed 30-s interval plus a random 120-s interval. A random ordering of the four stimuli was presented seven times followed by a random ordering of three stimuli, for a total of 31 stimulus presentations. The four stimuli (noise, clicker, houselight, and flashing light) were labeled as A, B, C, and D with the restriction that A and D were always drawn from a different modality than were $\mathrm{B}$ and $\mathrm{C}$. Thus, there were eight possible combinations of assignments of stimuli to the labels A, B, C, and D. Three rats were randomly assigned to each stimulus combination with consideration of their previous experience (the first from the first experiment, the second from the second experiment, and the third from the first or second experiment).

The 80 sessions were divided into four successive phases of 20 sessions each. In all four phases, Stimulus $\mathrm{C}$ was followed by the delivery of a food pellet $(\mathrm{C}+)$, and Stimulus D was not (D-). In Phases 1 and 3, Stimulus A was followed by a food pellet and Stimulus B was not (A+ and B-); in Phases 2 and 4, Stimulus A was not followed by a food pellet and Stimulus $\mathrm{B}$ was followed by a food pellet (A- and $\mathrm{B}+$ ).

At the end of some sessions, four 30-s compound stimuli without food were presented in the order $\mathrm{AB}, \mathrm{CD}, \mathrm{CD}, \mathrm{AB}$ or $\mathrm{CD}, \mathrm{AB}, \mathrm{AB}, \mathrm{CD}$, counterbalanced across rats. These occurred during Phase 2 on Sessions 2, 4, 5, and 15; during Phase 3 on Sessions 2, 6, and 15; and during Phase 4 on Sessions 4, 5, 6, and 7. One rat developed a tumor and was removed from the experiment after Phase 2. The data are shown, and statistics determined, for all 24 rats during Phases 1 and 2 and for 23 rats during Phases 3 and 4 .

\section{Results}

\section{Response Rate During Training}

Figure 1 (left) shows the mean response rate during the 30-s stimuli as a function of blocks of four cycles of training on each of the stimuli during Phases 2-4. (There were two blocks of four cycles of training on each of the stimuli on each session.) During Phase 1, the stimuli followed by food $(\mathrm{C}+$ and $\mathrm{A}+)$ led to a high response rate, and the stimuli not followed by food (B- and D-) led to a low response rate. During Phase 2, when the conditions of reinforcement changed for two of the stimuli (A and B), the response rate adjusted so that the response rate during the stimulus that had now changed to $\mathrm{B}+$ converged with the response rate during the stimulus that had remained as $\mathrm{C}+$; similarly, the response rate during the stimulus that had now changed to $\mathrm{A}-$ converged with the response rate during the stimulus that had remained as D-. Similar results occurred during Phases 3 and 4 in which the conditions of reinforcement continued to change for two of the stimuli (A and B).

A standard linear operator model (Equation 3 in the Discussion section) was applied to the mean response rate as a function of blocks of four cycles for the stimuli during which the reinforcement conditions changed between phases (A and B). For this purpose, $V(N)$ refers to the response rate on trial $N, \lambda$ refers to the asymptotic response rate, and $\beta$ refers to the learning rate. The learning rate parameters during acquisition and during extinction $\left(\beta_{a}\right.$ and $\left.\beta_{e}\right)$ and the asymptotic response rate parameters $\left(\lambda_{a}\right.$ and $\left.\lambda_{e}\right)$ were estimated using the NLINFIT function of Matlab 7.0. The rate of acquisition and extinction of responding were calculated and shown by the rising and falling smooth functions near the data of $\mathrm{A}$ and $\mathrm{B}$. The mean and standard error of the mean of $\beta_{a}$ in Phases 2, 3, and 4 of training were .066 (.007), .095 (.021), and .121 (.016), respectively; the mean and standard error of the mean of $\beta_{e}$ in Phases 2, 3, and 4 were .040 (.005), .023 (.002), and .030 (.006), respectively. A two-factor withinsubjects analysis of variance was calculated. The rate of acquisition was greater than the rate of extinction, $F(1,22)=33.58, p<.001$, and there was no effect of phase, $F(2,44)=1.89, p=.164$. There was an interaction of the reinforcement and phase factors, $F(2,44)=$ $4.83, p=.013$, which indicates that there was an increase in the difference between the rates of acquisition and extinction as a function of phase.

\section{Testing With Stimulus Compounds}

Figure 1 (right) shows the response rate during the compound stimuli ( $\mathrm{AB}$ and $\mathrm{CD}$ ) during Phases 2, 3, and 4 of testing. The response rate during the compound was averaged across compound stimuli presentations within a phase and averaged across rats. There was a temporal gradient of responding in which the response rate increased as a function of time since stimulus onset. The proportion of response gradients that increased in each of the three phases was greater than .95 (binomial tests, $p<.001$ ). A two-factor within-subjects analysis of variance was calculated. The slope of the response rate gradient during Stimulus AB was greater 
Training
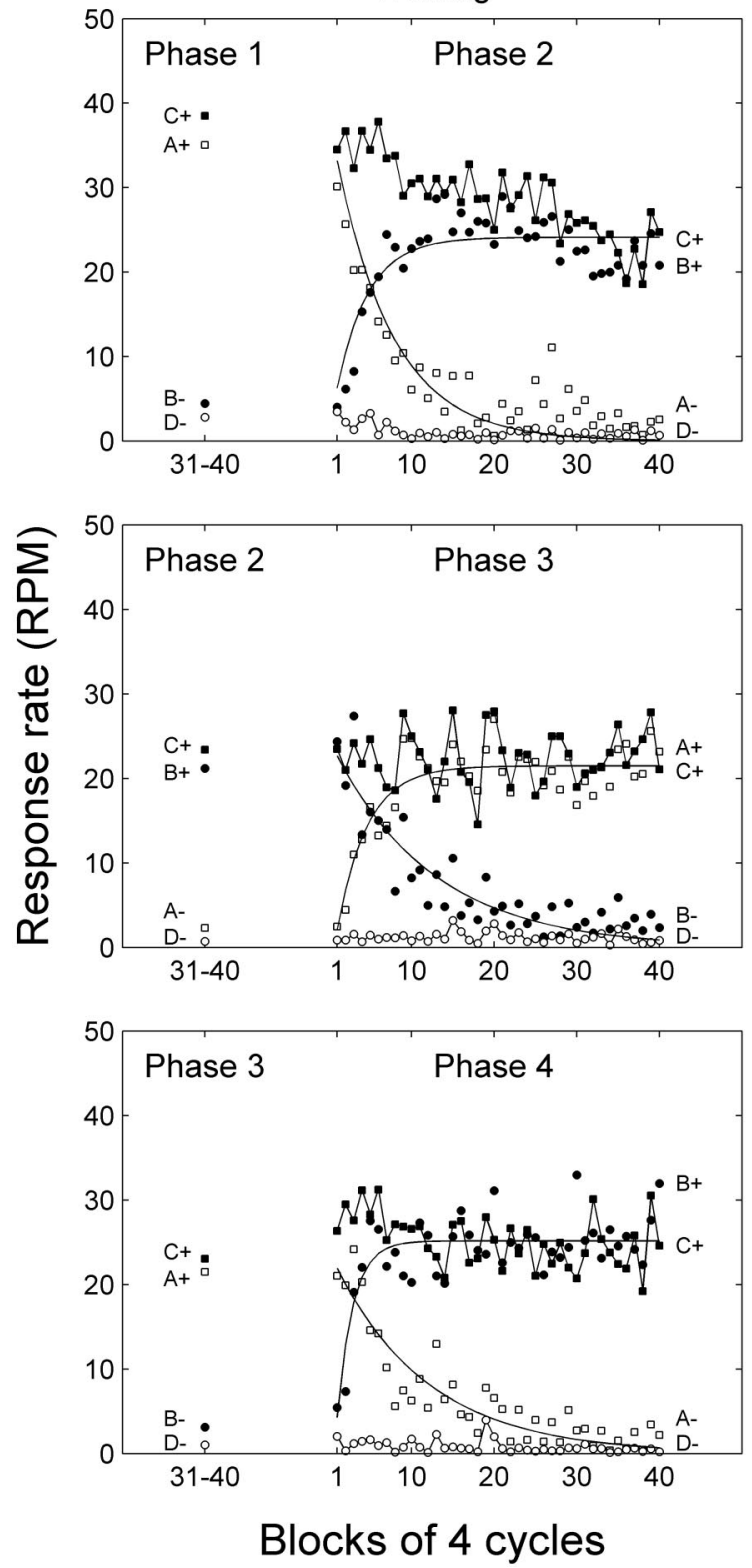

Testing
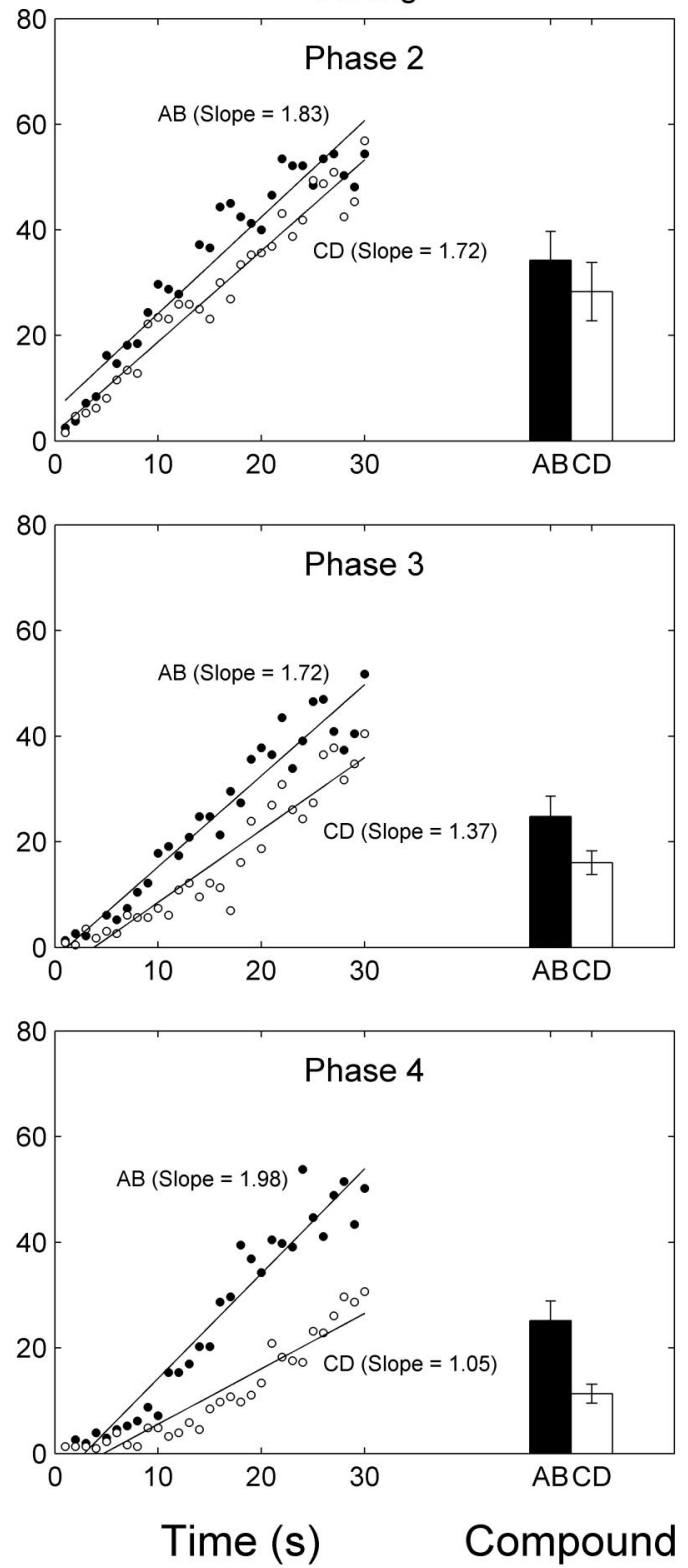

Figure 1. Training (left): Mean response rate during the 30-s presentation of the stimuli A, B, C, and D as a function of blocks of four cycles (half of a session). Testing (right): Mean response rate as a function of time since stimulus onset for compound stimuli $\mathrm{AB}$ and $\mathrm{CD}$. The rows are for phases.

than during Stimulus $\mathrm{CD}, F(1,22)=10.88, p=.003$, and there was no effect of phase, $F(2,44)=1.997, p=.148$. There was an interaction of the reinforcement and phase factors, $F(2,44)=$ $4.72, p=.014$, which indicates that the slopes became increasingly different in successive phases.

Figure 1 (right) also shows the mean response rate during the compound stimuli (AB and $\mathrm{CD}$ ) during the three phases. A twofactor within-subjects analysis of variance was calculated. The mean response rate was greater during Stimulus $\mathrm{AB}$ than during Stimulus CD, $F(1,22)=23.37, p<.001$; the mean response rate decreased in successive phases, $F(2,44)=11.72, p<.001$; but the interaction between condition and phase was not significant $F(2,44)=2.98, p=.061$.

\section{Response Gradients During Training}

Figure 2 shows the response rate as a function of time since stimulus onset for the four stimuli (A, B, C, and D) during the last five sessions of the last three phases of the experiment. The straight lines provide a rough estimate of the slope of the response gradients. 
Phase 2
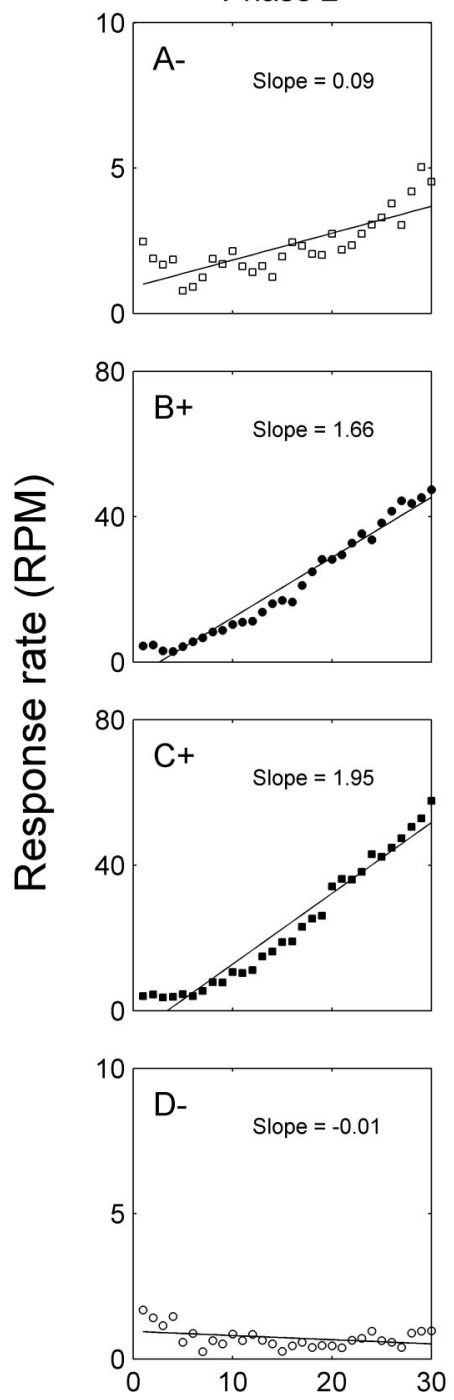

Phase 3
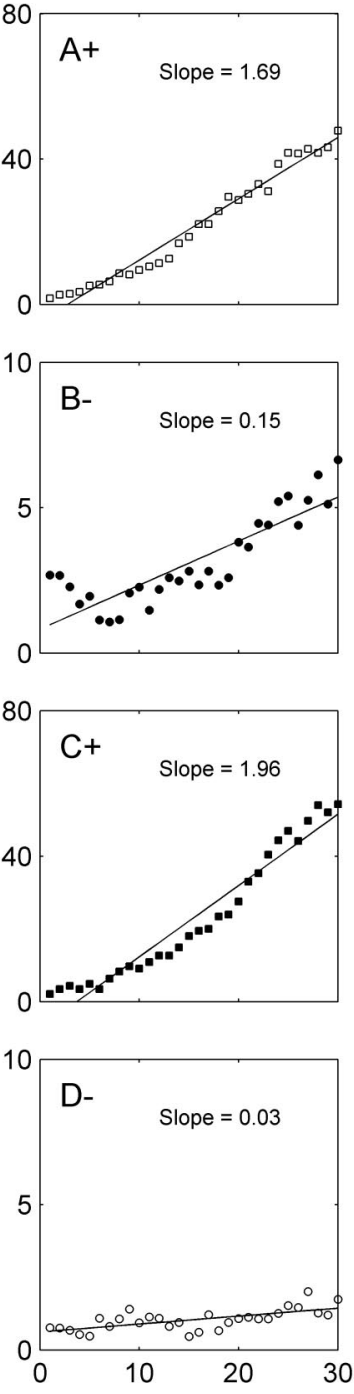

Time (s)
Phase 4
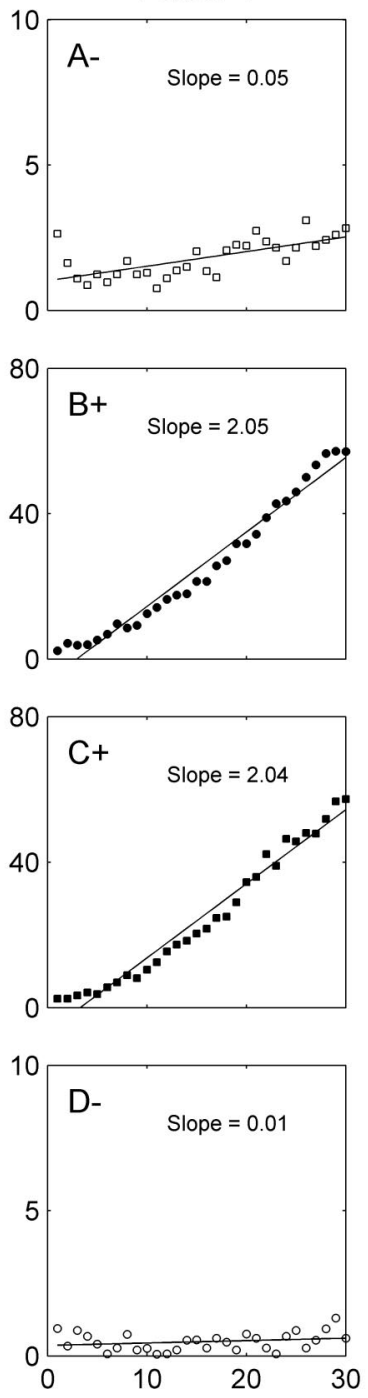

Figure 2. Mean response rate as a function of time since stimulus onset during the last five sessions of each phase. The rows are different stimuli (A, B, C, and D); the columns are different phases (2, 3, and 4). Note that the scale of acquisition $(+)$ is eight times the scale during extinction $(-)$.

The third row shows the response gradients for Stimulus $\mathrm{C}$ that was always followed by food; under this condition, the proportion of response gradients that increased in each of the three phases was 1.0 (binomial tests, $p<.001$ for each). The fourth row shows the response gradient for Stimulus D that was never followed by food; under this condition, all the response gradients were relatively flat. The proportion of response gradients that increased in the three phases was .46, .61, and .48 (binomial tests, $p=.58, .15$, and .58 , respectively). Note that the response rate scale is eight times higher for acquisition conditions $(+)$ than for extinction conditions (-).

The first two rows show the response gradients for Stimuli A and $\mathrm{B}$ that alternated between reinforcement and nonreinforcement on successive phases. The general rule was that a response gradient developed to a stimulus that was followed by food, $\mathrm{A}+$ and $\mathrm{B}+$, and it remained during stimuli that had been followed by food, but which were not currently being followed by food, A- and B-. The proportion of response gradients that increased for Stimulus A in the three phases were $.75,1.0$, and .70 (binomial tests, $p=.003$, $p<.001$, and $p=.032$ ); the proportion of response gradients that increased for Stimulus B in the three phases were 1.0, .78, and 1.0 (binomial tests, $p<.001$ in all cases; note that food followed the 30-s presentations of Stimulus A in Phase 1).

\section{Discussion}

In the magazine approach procedure, rats readily learned the stimulus discrimination to the two auditory and two visual stimuli, 
and the reversals between the conditions of acquisition and extinction.

\section{Effects of Conditions on Response Rate}

During each of the four phases, the response rate in the presence of the two stimuli that were followed by food remained at, or were increased to, a high steady rate, and the response rate in the presence of the two stimuli that were not followed by food remained at, or were reduced to, a low steady rate. The application of the standard linear stochastic model of learning and extinction to the response rates led to the conclusion that the mean rate of acquisition parameter $\left(\beta_{a}=.094\right)$ was greater than the mean rate of extinction parameter $\left(\beta_{e}=.031\right)$. A similar conclusion came from the comparison of the response rate of the compound stimulus $(\mathrm{AB})$ that was composed of the two stimuli that were alternated between acquisition and extinction between phases, and the compound stimulus (CD) that was composed of a stimulus that was always followed by food and one that was never followed by food. The response rate was greater in the $\mathrm{AB}$ compound than in the $\mathrm{CD}$ compound. This indicates that presentations of stimuli followed by food increased the response rate more than the same number of presentations of stimuli that were not followed by food decreased the response rate.

A stimulus $(\mathrm{C}+)$ that was always followed by food after $30 \mathrm{~s}$ produced temporal conditioning; that is, response rate increased as a function of time since stimulus onset. Another 30-s stimulus (D-) that was never followed by food did not show any temporal conditioning; that is, the response rate was relatively constant after stimulus onset. By the end of any phase in which a stimulus was followed by food after $30 \mathrm{~s}(\mathrm{~A}+, \mathrm{B}+$, and $\mathrm{C}+)$, there was temporal conditioning. During a subsequent extinction phase (Aor B-), the effects of temporal conditioning were still present, although the response rate was lowered. Thus, the presentation of a stimulus without food (i.e., an extinction procedure) reduced response rate to a relatively constant low level, but it did not change the previously acquired pattern of responding. In successive phases, the rate of acquisition increased more than the rate of extinction. In standard linear models of learning, the learning parameters are held constant throughout training, although learning sets occur under many conditions (Fagan \& Olton, 1987; Harlow, 1949; Slotnick, Hanford, \& Hodos, 2000).

With the addition of a linear operator rule to packet theory, quantitative predictions can be made not only about asymptotic performance (Guilhardi et al., 2005; Kirkpatrick, 2002; Kirkpatrick \& Church, 2003) but also about the acquisition (Guilhardi \& Church, 2005) and extinction (Guilhardi \& Church, in press) of response rate and pattern. These predictions about behavior can be evaluated with a Turing test (Church \& Guilhardi, 2005). These, however, are predictions about the behavior, and not necessarily about associative strength.

\section{Effects of Conditions on Associative Strength}

The standard linear model of learning, written in the notation of the Rescorla and Wagner (1972) model is

$$
\Delta V(N)=\alpha \beta(\lambda-V(N))
$$

$$
\Delta V(N)=V(N+1)-V(N)
$$

where $V$ is associative strength, $N$ is the cycle number, $\alpha$ is the learning rate parameter associated with the conditioned stimulus (CS), $\beta$ is the learning rate parameter associated with the unconditioned stimulus (US), and $\lambda$ is the asymptote of learning supported by the US. Equations 1 and 2 are linear difference equations over $N$, and the solution is

$$
V(N)=\lambda+(1-\alpha \beta)^{N-1}\left(V_{0}-\lambda\right)
$$

(see, e.g., Boyce and DiPrima (2005), equation 15, p. 122).

Let $V_{A}, V_{B}, V_{C}$, and $V_{D}$ denote the strength associated with Stimulus A, B, C, and D, respectively. Because those stimuli are counterbalanced, and assumed to be equally salient, the learning parameters associated with CSs are unbiased and can be simplified as $\alpha_{A}=\alpha_{B}=\alpha_{C}=\alpha_{D}=\alpha$.

The subscripts $a$ and $e$ will be used to label acquisition and extinction, respectively. Here, the asymptote of acquisition $\lambda_{a}$ is set as 1 , and the asymptote of extinction $\lambda_{e}$ is set as 0 . To further simplify equations, let us define the following variables:

$$
b_{a}=1-\alpha \beta_{a}
$$

$\beta_{a}$ is an acquisition rate parameter associated with US

$$
b_{e}=1-\alpha \beta_{e}
$$

$\beta_{e}$ is an extinction rate parameter associated with US

$$
n=N-1
$$

$N$ is a cycle number in Phase 1

$$
m=M-1
$$

$M$ is a cycle number in Phase 2

According to Equation 3, at the end of Phase 1 (after $N$ cycles), the associative strengths are as follows.

$$
\begin{gathered}
V_{A}(N)=1+b_{a}^{n}\left(V_{0}-1\right) \\
V_{B}(N)=b_{e}^{n} V_{0} \\
V_{C}(N)=1+b_{a}^{n}\left(V_{0}-1\right) \\
V_{D}(N)=b_{e}^{n} V_{0}
\end{gathered}
$$

At the end of Phase 2 (after another $M$ cycles), the associative strengths are as follows.

$$
\begin{gathered}
V_{A}(M)=b_{e}^{m} V_{A}(N) \\
V_{B}(M)=1+b_{a}^{m}\left(V_{B}(N)-1\right) \\
V_{C}(M)=1+b_{a}^{m}\left(V_{C}(N)-1\right) \\
V_{D}(M)=b_{e}^{m} V_{D}(N)
\end{gathered}
$$

According to the Rescorla-Wagner model, the associative strength of a compound stimulus is the summation of the strength of each individual stimulus (Rescorla \& Wagner, 1972). Thus, following the argument developed by Rescorla (2002), the associative strength of compound stimuli $\mathrm{AB}$ is 
$V_{A B}=V_{A}(M)+V_{B}(M)=b_{e}^{m}\left[b_{a}^{n}\left(V_{0}-1\right)+1\right]$

$$
+b_{a}^{m}\left(b_{e}^{n} \cdot V_{0}-1\right)+1
$$

and the associative strength of compound stimuli $\mathrm{CD}$ is

$V_{C D}=V_{C}(M)+V_{D}(M)=b_{a}^{m} \cdot b_{a}^{n}\left(V_{0}-1\right)+1+b_{e}^{m} \cdot b_{e}^{n} \cdot V_{0}$.

To compare the associative strengths of $\mathrm{AB}$ and $\mathrm{CD}, V_{C D}$ is subtracted from $V_{A B}$. After rearrangement, Equation 14 follows:

$$
V_{A B}-V_{C D}=\left(b_{e}^{m}-b_{a}^{m}\right)\left[1-b_{a}^{n}+V_{0}\left(b_{a}^{n}-b_{e}^{n}\right)\right] .
$$

Because $0<b_{a}<1$ and $0<b_{e}<1$ and, at the end of Phase 1, the associative strengths are close to asymptote, $n$ (or $N$ ) should be large enough for both $b_{a}^{n}$ and $b_{e}^{n}$ to be close to 0 . Thus, the difference between the strengths of $\mathrm{AB}$ and $\mathrm{CD}$ are close to $b_{e}^{m}-b_{a}^{m}$, which can be rewritten as

$$
V_{A B}-V_{C D}=\left(1-\alpha \beta_{e}\right)^{M-1}-\left(1-\alpha \beta_{a}\right)^{M-1} .
$$

Based on Equation 15, we can predict as follows:

1. On the first extinction cycle $(M=1)$, the value of Equation 15 is zero, so there will be no difference between the strength of $\mathrm{AB}$ and the strength of $\mathrm{CD}$.

2. At asymptote $(M \rightarrow \infty)$, the value of Equation 15 approaches zero, so there will also be no difference between the strength of $\mathrm{AB}$ and the strength of $\mathrm{CD}$.

3. At moderate values of $\mathrm{M}$,

a. if $\beta_{a}=\beta_{e}$, the value of Equation 15 is zero, which means there will be no difference between the strength of $\mathrm{AB}$ and the strength of $\mathrm{CD}$;

b. if $\beta_{a}>\beta_{e}$, Equation 15 is positive, which means the strength of $\mathrm{AB}$ is greater than that of $\mathrm{CD}$;

c. if $\beta_{a}<\beta_{e}$, Equation 15 is negative, which means the strength of $A B$ is less than that of $C D$.

The logic of the experiment design used by Rescorla (2002) was based on four assumptions: (a) a basic linear operator model of acquisition and extinction of associative strength; (b) the summation combination rule in which the associative strength of a compound stimulus is equal to the sum of the associative strength of its elements; (c) an ordinal relationship between response rate and associative strength; and (d) an equivalent, or negligible, extinction of the unreinforced compound stimuli.

\section{Relationship Between Response Rate and Associative Strength}

Rescorla (2002) assumed that there is an ordinal relationship between response rate and associative strength, which may be called an ordinal mapping rule. Thus, response rate can be expressed as a function of associative strength as follows:

$$
R(N)=f(V(N)),
$$

where $f(x)$ is a monotonic function. Here, let us arbitrarily assume the mapping rule to be a generalized power function:

$$
R(N)=K \cdot V(N)^{p}+R_{0}
$$

where the coefficient $K$ and the power $p$ are positive constants, and the baseline of response rate $R_{0}$ is nonnegative. By substitution of Equation 3 into Equation 17, Equation 18 is obtained, in which response rate is expressed as a function of cycle number $N$ :

$$
R(N)=K \cdot\left[\lambda+(1-\alpha \beta)^{N-1}\right]^{p}+R_{0} .
$$

In Equation 18, there are four free parameters: the coefficient $K$, the learning parameter $\alpha \beta$, the baseline $R_{0}$, and the power $p$. Those parameters were estimated by using NLINFIT in Matlab 7.0, which uses the Gauss-Newton algorithm. The median values were $K=22, \alpha \beta_{a}=.10, \alpha \beta_{e}=.04, R_{0}=3$, and $p=1.0$. The fact that the best fitting power was close to 1.0 implies that the relationship between response rate and associative strength was approximately linear.

\section{Conclusion}

Based on the transfer design, with a higher response rate in the $\mathrm{AB}$ compound than in the $\mathrm{CD}$ compound, and the logic described by Rescorla (2002), the present experiments support the conclusion that associative strength is increased more by the presentation of a stimulus followed by food than it is decreased by the presentation of a stimulus that is not followed by food. There are four assumptions: (a) the linear operator rule for associative strength (Equation 1), (b) the summation combination rule for the associative strength of a compound stimulus (Equations 12 and 13), (c) the ordinal relationship between response rate and associative strength, and (d) the equivalent, or negligible, extinction of $\mathrm{AB}$ and $\mathrm{CD}$. With these assumptions, and the observed response rates during the stimulus elements and compounds, the mapping rule between response rate and associative strength can be estimated. Equation 18 considers all simple power function transformations (Tukey, 1977) and concludes that the exponent is approximately 1.0. This suggests that the relationship between response rate and associative strength is approximately linear in this experiment.

For the goal of prediction of response rate (rather than of associative strength), none of these assumptions are required because it is possible to observe the degree of fit of the linear operator rule of the response rate and the fit of any combination rule from the response rate in two elementary stimuli to the response rate in a compound stimulus with these two elements. With the goal of predicting response rate, it is possible to estimate the magnitude of the increments produced by the presentation of food at the end of a stimulus and the magnitude of the decrements produced by the presentation of a stimulus without food (rather than simply the ordinal distinction between these two values).

In addition to the acquisition and extinction of the response rate, this procedure also produced substantial and regular temporal gradients and changes in these gradients as a function of training. The rates of acquisition and extinction of temporal gradients were quite different than for response rates. In this experiment, and in others (Guilhardi \& Church, in press; Ohyama et al., 1999), the extinction operation decreased the response rate profoundly but had only a minimal effect on the response pattern.

\section{References}

Boyce, W. E., \& DiPrima, R. C. (2005). Elementary differential equations and boundary value problems (8th ed.). Hoboken, NJ: Wiley. 
Bush, R. R., \& Mosteller, F. (1951). A mathematical model for simple learning. Psychological Review, 58, 313-323.

Church, R. M., \& Guilhardi, P. (2005). A Turing test of a timing theory. Behavioural Processes, 69, 45-58.

Estes, W. K. (1950). Toward a statistical theory of learning. Psychological Review, 57, 94-107.

Fagan, A. M., \& Olton, D. S. (1987). Spatial learning set acquisition in rats. Quarterly Journal of Experimental Psychology: Comparative and Physiological Psychology, 39(B), 65-75.

Guilhardi, P., \& Church, R. M. (2005). Dynamics of temporal discrimination. Learning \& Behavior 33, 399-416.

Guilhardi, P., \& Church, R. M. (in press). The pattern of responding after extensive extinction. Learning and Behavior.

Guilhardi, P., Keen, R., MacInnis, M. L. M., \& Church, R. M. (2005). How rats combine temporal cues. Behavioural Processes, 69, 189-205.

Harlow, H. F. (1949). The formation of learning sets. Psychological Review, 56, 51-65.

Kirkpatrick, K. (2002). Packet theory of conditioning and timing. Behavioural Processes, 57, 89-106.

Kirkpatrick, K., \& Church, R. M. (2003). Tracking of the expected time to reinforcement in temporal conditioning procedures. Learning \& Behavior, 31, 3-21.

Ohyama, T., Gibbon, J., Deich, J. D., \& Balsam, P. D. (1999). Temporal control during maintenance and extinction of conditioned keypecking in ring doves. Animal Learning \& Behavior, 27, 89-98.

Rescorla, R. A. (1996). Preservation of Pavlovian associations through extinction. Quarterly Journal of Experimental Psychology: Comparative and Physiological Psychology, 49(B), 245-258.

Rescorla, R. A. (2000). Associative changes in exciters and inhibitors differ when they are conditioning in compound. Journal of Experimental Psychology: Animal Behavior Processes, 26, 428-438.

Rescorla, R. A. (2001a). Experimental extinction. In S. B. Klein and R. R. Mowrer (Eds.), Handbook of contemporary learning theories. Mahwah, NJ: Lawrence Erlbaum Associates, pp. 119-154.

Rescorla, R. A. (2001b). Unequal associative changes when exciters and neutral stimuli are conditioned in compound. Quarterly Journal of Experimental Psychology: Comparative and Physiological Psychology, 54(B), 53-68.

Rescorla, R. A. (2002). Comparison of the rates of associative change during acquisition and extinction. Journal of Experimental Psychology: Animal Behavior Processes, 28, 406-415.

Rescorla, R. A., \& Wagner, A. R. (1972). A theory of Pavlovian conditioning: Variations in the effectiveness of reinforcement and nonreinforcement. In A. H. Black \& W. F. Prokasy (Eds.), Classical conditioning II: Current research and theory (pp. 64-99). New York: Appleton-Century-Crofts.

Slotnick, B., Hanford, L., \& Hodos, W. (2000). Can rats acquire an olfactory learning set? Journal of Experimental Psychology: Animal Behavior Processes, 26, 399-415.

Tatham, T. A., \& Zurn, K. R. (1989). The Med-PC experimental apparatus programming system. Behavioral Research Methods, Instruments, \& Computers, 21, 294-302.

Tukey, J. W. (1977). Exploratory data analysis. Reading, MA: Addison Wesley.

Received September 20, 2005

Revision received December 13, 2005

Accepted December 13, 2005

\section{E-Mail Notification of Your Latest Issue Online!}

Would you like to know when the next issue of your favorite APA journal will be available online? This service is now available to you. Sign up at http://watson.apa.org/ notify/ and you will be notified by e-mail when issues of interest to you become available! 